\title{
EL DERECHO PROTECCIÓN Y LA REGULACIÓN DEL USO DE LAS NUEVAS TECNOLOGÍAS DESDE EL DERECHO ADMINISTRATIVO
}

\author{
A CORRETA PROTEÇÃO E REGULAMENTAÇÃO DO USO DE NOVAS \\ TECNOLOGIAS DO DIREITO ADMINISTRATIVO
}

\author{
THE RIGHT PROTECTION AND REGULATION OF THE USE OF NEW \\ TECHNOLOGIES FROM THE ADMINISTRATIVE LAW
}

\author{
NANCY Nelly GonzÁLEZ SANMiguel \\ nancygonsa09@hotmail.com \\ Universidad Autónoma de Nuevo León. \\ Nuevo León, México.
}

\begin{abstract}
RESUMEN
El uso de la tecnología es una revolución en la que se encuentra inmersa la sociedad y como parte de este hecho, el Derecho tiene la necesidad de su regulación y a su vez incorporar su aplicación dentro de sus actividades, principalmente dentro del Derecho Administrativo, mismo que deberá adecuarse e implementar un cambio hacia un gobierno electrónico que considere los principios que se deben aplicarse para lograr consolidar la noción de una Buena Administración y a su vez tener una eficientización dentro sus procesos y actos administrativos, siendo estos factores determinantes para hacia una tecnoadministración.
\end{abstract}

Palabras clave: Derecho, Administración; Tecnología; Regulación; Acto Administrativo.

\begin{abstract}
The use of technology is a revolution that society is experiencing and as part of it, the Law has the need for its regulation and in turn to its application within its activities, particularly within the Administrative Law that will have to be implement a change towards an electronic government, which considers the principles that must be considered to carry the concept of a Good Administration and at the same time have an efficiency within its administrative processes and acts, being these determining factors towards a technoadminitration.
\end{abstract}

Keywords: Law, Administration; Technology; Regulation; Administrative Act.

\section{RESUMO}

O uso da tecnologia é uma revolução que a sociedade está experimentando e, como parte dela, a Lei precisa de sua regulamentação e, por sua vez, de sua aplicação em suas atividades, particularmente no Direito Administrativo que deverá ser implementar uma mudança em direção a um governo eletrônico, que considere os princípios que devem ser considerados como portadores do conceito de Boa Administração e, ao mesmo tempo, ter eficiência em seus processos e atos administrativos, sendo esses fatores determinantes para uma tecnoadminitração.

Palavras-chave: Direito, Administração; Tecnologia; Regulamento; Lei Administrativa.

\section{SUMÁRIO}

INTRODUCCIÓN; 1.-Derecho Público y las nuevas tecnologías; 1.1 Regulación del Derecho a la Información y el Derecho Administrativo; 1.2 Tecnología; 1.3 Aspectos Positivos o Negativos con el uso de la tecnología dentro de la Administración Pública; 2.- Transparencia de la Información; 3.Principios que debe contar la Administración Pública Electrónica; 4.- La efectividad del acto administrativo en la vía tecnología; 4.1 Conceptualización del Acto Administrativo; CONCLUSIÓN; REFERENCIAS. 


\section{INTRODUCCIÓN}

El presente trabajo de investigación versa acerca del cambio de que ha sufrido la sociedad debido a la implementación de la tecnología, así como la regulación de esta dentro del Derecho y en particular con los actos que son emitidos por la Administración Pública, teniendo un impacto dentro de la estructura jurídica y visualizando la manera de priorizar la eficiencia de la Administración.

Dentro del mismo se señalarán las visiones que tiene el derecho público con la revolución de la tecnología dentro del Derecho, así como su aplicación en el Derecho Administrativo, señalando aquellos aspectos tanto positivos o negativos en la utilización de la tecnología dentro del Derecho y los cambios para la manifestación de la voluntad de los sujetos a través de los medios electrónicos.

Se constituyen los principios que se deben observar dentro de la Administración Pública, tales como la seguridad, la igualdad, la letalidad, la conservación de las garantías, la realización de las actuaciones con trasparencia y de proporcionalidad por el uso de digital dentro de sus actuaciones.

El objetivo de este trabajo de investigación es relacionar el avance del uso de la tecnología con la sistematización del proceso de los actos administrativos de la Administración Pública y cómo se ha ido avanzando en esta materia dentro de la normatividad en México, pues a pesar de que no se contemplan estos lineamientos se han ido incorporando nuevos elementos como la firma electrónica y documentos digitales.

Considerando la importancia de esta incursión de la efectividad de los actos administrativo para llevar el uso de la tecnología para dar una efectividad y a su vez que sean validas dichas actuaciones, siendo un cambio hacia un gobierno digital que se considera dentro de los principios de Transparencia hacia la era de un Buena Administración. Para ello se utilizó el método analítico ya que se realizó una exhaustiva búsqueda de información para después comparar y establecer los puntos más relevantes.

\section{DERECHO PÚBLICO Y LAS NUEVAS TECNOLOGÍAS}

El Estado tiene que adecuarse a los efectos que produce la tecnología incorporándola y modificando los procesos que anteriormente se utilizaban, los cuales están facultados por la 
norma jurídica para sistematizar varios procesos, para contribuir a una mayor aproximación con el ciudadano por medio del uso de redes.

La conexión que surge entre el Estado y los particulares a través del uso de la tecnología se da debido a que las "nuevas tecnologías se vuelven un factor considerable en la acentuación de los fenómenos de dominación, de concentración y centralización de las capacidades de decisión y acción, en favor del estado y el sector público, y/o de las corporaciones y consorcios privados"1. El Estado se ha ajustado a la nueva forma de cómo se actúa dentro de la relación jurídica, modificando de esta manera cómo versa la posición del Estado en la redefinición de los procesos de sus funciones con las nuevas tecnologías.

El cambio que está sufriendo la sociedad por la implementación de los avances tecnológicos y en particular por el uso de las tecnologías en el campo del Derecho Administrativo es lo que se denomina tecnoburocracia, dando pauta con esto a la incorporación de la tecnología a través de las normas jurídicas para llevar acabo los actos de la Administración, teniendo como fin una mayor transparencia dentro de los procesos en las Administración Pública.

La incorporación de las tecnologías en las normas ha representado un impacto en la forma organizativa del Derecho Administrativo, teniendo que relacionar la responsabilidad de este con la innovación en los procesos, teniendo que dirigirse a un cambio de paradigmas debido a la conexión tan rápida de la información, eficientizando ciertos tramites y también dando la oportunidad de que se transparenten las acciones del Estado a través de la publicación digital de ciertos informes.

El uso de la tecnología se ha ido positivizando para permitir usarla dentro de la Administración Pública, y que con ello se genere un impacto positivo dentro de la misma, asumiendo cambios en la forma de cómo se realiza la organización y la producción de los actos jurídicos, modificando el concepto de la soberanía del Estado.

Considerando que las normas jurídicas no son a veces adecuadas al impacto de la tecnología como para entrar en el Derecho, tentemos ahora que la Revolución Tecnología se ha manifestado a través del Derecho y en lo que nos incumbe dentro del Derecho Administrativo, mediante cambios dentro de la actuación de procesos, de actos administrativos, de manera que se puede considerar por una parte el énfasis en la eficacia y por otra se puede considerar que no se tiene una comunicación con la Autoridad Administrativa.

\footnotetext{
${ }^{1}$ Kaplan, Marcos. Ciencia, Estado y derecho en la tercera Revolución. Universidad Autónoma de México, 2000, México, pág. 213.
} 
Los nuevos acontecimientos de la ciencia generan cambios y exigen reacciones que se tienen que ver reflejadas en la agilización del derecho para estar a la par de la competencia y evitar crisis dentro de la modernización del Derecho. Constituye un factor muy importante del Estado definir cómo poder utilizar la tecnología dentro de su ámbito, considerando que las “crecientes funciones de intervención, de regulación, de control, incluso un papel económico directo, todo ello con un grado creciente de rectoría. Las funciones del Estado se van ampliando sucesiva y permanentemente, dando lugar a un redefinición cuantitativa o cualitativa de la naturaleza de aquel, de su papel, del carácter de sus actividades"2. El Derecho tiene que aplicar los cambios que tanto la sociedad como la tecnología sufren para poder regularizar y aplicar el mismo dentro de nuestro campo.

Puede visualizarse que el Estado logra permitir que ciertos actos se realicen por medio del uso de la tecnología ya sea por ejemplo por la presión de la sociedad, los cuales tiene que estar previamente previstos por una norma legal para lograr que las políticas públicas alcancen una mejor cercanía y efectividad para dirimir las necesidades de una manera más rápida y congruente.

El Estado a través de la función pública se configura como aquel que forma parte del proceso socioeconómico del país siendo esta una tarea de gran importancia, por lo que debe procurar estar acorde con los demás Estados y tener un equilibrio entre el Derecho y las necesidades que se presentan dentro de la sociedad, promoviendo el cambio hacia el uso de la tecnología dentro de estos campos.

Considerando que la tecnología puede cambiar el medio de los instrumentos y los mecanismos estatales como la Administración Pública al realizar sus actividades debido al cambio de uso de nuevos dispositivos mismos que el Derecho ha regulado por disposiciones jurídicas para estar acorde a las situaciones que se viven en la actualidad.

Es así como la ciencia, en particular la tecnológica se ha visto que ha influido dentro del orden social, dando un cambio de las formas de actuar tanto de los ciudadanos como de la Administración Pública, marcando una transformación dentro del dinamismo de sus actuaciones, adaptando ciertas medidas para establecer la tecnología dentro de sus procesos y así poder redistribuir sus funciones y actividades, manifestando con esto el término de la tecno administración debido a la tecnificación de las actividades administrativas que se están presentando dentro del Estado.

\footnotetext{
${ }^{2}$ Kaplan, Marcos. Ciencia, Estado y derecho en la tercera Revolución. Universidad Autónoma de México, 2000, México. p. 146
} 


\subsection{Regulación del Derecho a la Información y el Derecho Administrativo}

El uso de las nuevas tecnologías da un parámetro para contar con mayor transparencia e información por parte de la Administración Pública, es así que ahora la comunidad se puede percatar de los actos de la Administración Pública de una manera instantánea debido a esto, al igual que al solicitar una información o trámite se agiliza por la implementación del uso de computadoras, internet, plataformas que son amigables para la agilización en la búsqueda de datos y cumplir en ciertos casos obligaciones que se generan dentro de la Administración o solicitar algo de esta.

Se ha constituido que a raíz de esta apertura del Derecho Informático y el uso de las nuevas tecnologías es posible constituir un Estado de Derecho basado en el establecimiento de parámetros de las garantías para contemplar este tipo de uso dentro de las libertades que tienen los gobernados y a su vez otorgarles la oportunidad de tener una participación más democrática debido a que las necesidades se van comunicado a través de estos medios.

Contemplando que la Administración Pública forma parte del Estado y a través de esta se realizan las actividades de los órganos, siendo la Administración quien constituye los instrumentos para realizar el objetivo de culminar con las necesidades de los ciudadanos, en la forma que actualmente estamos viviendo debido a los cambios paradigmas, se ha visto que unos de los mayores cambios que han traído los usos tecnológicos es la demarcación de los espacios, pues gracias a las tecnologías se van desaparecieron los espacios físicos debido a que ahora lo que allí se realizaba se puede realizar ahora dentro de un plataforma y no en un lugar físico de ese órgano de la Administración, de manera que ahora el interesado podrá acceder de una manera más fácil y con mayor comodidad para externar la información solicitada.

Las funciones y las actividades que se realizan se han modificación debido a la implementación de uso de las tecnologías y acerca del Derecho Administrativo con el Derecho Informático se constata que para modificar y acceder a este tipo de medios tendrán que estar contenidas dentro de una norma jurídica para que tenga validez.

La Administración Pública funge un papel muy importante dentro del Estado debido a que en su constitución forma parte de los intereses de las decisiones que se tomen y esta tiene que cumplir con el objetivo máximo que es una regulación en la organización de la sociedad y esta debe irse ajustando a las necesidades evidente de la población.

El Estado teniendo que alcanzar dichos objetivos, destinados a cumplir con las necesidades de sus habitantes, debe de estar alerta ante la presencia de crisis, las cuales deberá 
afrontar a través de la autentificación de las normas jurídicas, de tal manera que estas señalen los criterios a seguir para dar una ventana de oportunidad de cambio, propiciando la transparencia y la confianza de los ciudadanos hacia a la Administración.

Sabemos que por naturaleza el hombre va cambiando o transformando el lugar donde habita y por la tanto la Administración Pública "debe ser promotora de los cambios sociales o adecuar el funcionamiento de sus órganos a los requerimientos de esos cambios, a efecto de que cumpla con la sociedad civil en la solución de los problemas que la misma le plantea a través de la historia"3.

Considerando como un concepto tanto de acercamiento como de modernización el uso de las tecnologías dentro de la Administración Pública, se debe realizar una modificación dentro de los preceptos legales para incrustar estos procesos o formas dentro de los actos que emite la Administración cumpliendo con las características que se deben seguir por parte de la Administración y así fijarse dentro de un régimen jurídico que tenga eficacia y validez.

Considerando que los medios tecnológicos sean utilizados para eficientizar las actividades o las funciones de la Administración, se implica con esto una disminución de costo en la reducción de ciertos materiales como el papel, entre otros debido a que las comunicaciones se pueden hacer a través de los medios electrónicos y el costo de los gastos de la Administración se reducen para utilizar dichos ingresos en otro tipo de necesidades.

Estableciendo que uno de los objetivos dentro de la Administración es proporcionar la mejor satisfacción hacia los gobernados de una manera adecuada y eficiente, utilizar los insumos de una manera correcta, ya sean materiales, financieros y humanos para culminar con sus funciones y obtener el resultado esperado que se necesita para lograr obtener los resultados de su gestión y a la vez fomentar el cuidado del medio ambiente en la utilización de otros medios de recursos que no afecten al ambiente, se genera una sistematización dentro de la Administración y un acercamiento más factible con los gobernados.

\subsection{Tecnología}

El uso de la tecnología es una transformación en nuestra vida cotidiana provocando un cambio dentro de la actuación de la sociedad, mismo que se ha regulado por el Derecho en específico en el Derecho Administrativo, siendo un cambio la introducción de este método en las actuaciones de este.

${ }^{3}$ Galindo Camacho, Miguel. Teoría de la Administración Pública. Porrúa, México, 2000. p. 114. 
Se pude observar cómo se ha introducido la tecnología dentro del Derecho Administrativo mediante la implementación de programas a través del uso de las tecnología en ciertas actividades, ejerciendo el cambio hacia un derecho electrónico, se observa que durante el año 2000 en México se constituye el Sistema de CompraNeta el cual se utilizaba para realizar la adquisición de Obras Pública, sistema que se encontraba reglamentado en la Ley de Obras Públicas y Servicios y la de Arrendamientos Adquisiciones y Servicios de Sector Público. Uno de los objetivos era dar facilidad y transparencia a los mismos, dando con esto un giro dentro de los contratos, ya que se procedió a realizarse en forma electrónica, para esto se fundamentó dentro de la Ley Federal de Procedimiento Administrativo el 30 de mayo del 2000, que corrobora la validez de los mismos pero señala que dichos contratos se tendrían que firmar de puño y letra, pues aún no se daba el cambio de la firma electrónica, sin embargo fueron los principios del cambio de la Administración Pública.

Dando con esto el comienzo de la era electrónica dentro de la Administración Pública debido a que través de los años se fueron incrementando las áreas que se iban implementando este tipo de sistemas. El 04 junio de 2002 se expidió la norma oficial mexicana 151-SCFI-2002 que establece la regla de la conservación de los mensaje de datos, así como se puede observar que dentro del año 2004 el 05 de enero se presentó la reforma al Código Fiscal de la Federación para la implantación de los medios electrónicos, dentro de ese mismo año en mayo se publica la resolución miscelánea fiscal que reconoce a los certificados de firma electrónica avanzada y a la facturación electrónica, siendo aquí el punto de partida de la revolución dentro de la Administración, comenzando con esto a permitir que ciertos tramites se efectuarán de la misma forma que sería dentro de varios formatos del Registro Federal de Contribuyentes, así como los reglas para la prestadores de aquellos que llevaran los servicios de certificación.

Las interacciones con el comercio electrónico se fueron reconociendo y a través de la Secretaría de Económica en relación con la Procuraduría Federal de Protección al Consumidor se introdujeron los principios de cómo se debe realizar los actos de comercio, dando con esto el paso al uso de medio electrónicos a través de la tecnología y ejerciendo un cambio de la relación jurídica.

El cambio de este paso, externó un nuevo paradigma de cómo se debe considerar la validez de dichos actos, así como la formalidad, debido a que se incorporó a la firma electrónica, dando validez a la firma autógrafa para que surtiera efectos dichos actos, dando ese paso al cambio de ciertos actos administrativos a la utilización de la tecnología con efectos jurídicos. 
La Firma electrónica se considera por la UNCITRAL como "los datos en forma electrónica consignados en un mensaje de datos o adjuntados o lógicamente asociados al mismo, que puedan ser utilizados para identificar al firmante en relación con el mensaje de datos e indicar que el firmante aprueba la información recogida en el mensaje de datos", dando con esto una autentificación de los actos, ya que se identifica quien los esta emitiendo, dando para mayor seguridad la imposición del sello digital que es lo que acredita que dicho documento fue recibido a la persona que se dirigió.

Se debe tener dentro de la sociedad un equilibrio entre el uso de la tecnología y la aplicación de esta dentro de la Administración ya que eso implica una amplitud y con esto se generan dos situaciones desde el aspecto positivo. Un aspecto positivo es que los gobernados pueden vigilar las acciones de la Administración y la utilización de los ingresos para el gasto público pero a la vez esa oportunidad que tenemos como gobernados también la administración puede ejercer un mayor control hacia nosotros dentro de nuestras obligaciones, por lo tanto lo que se debe de tutelar es una protección hacia esa libertad informática y establecer los límites y garantías de la misma para condicionar una estabilidad en las acciones del órgano del Estado y de los gobernados integrando esto con los derechos humanos.

\subsection{Aspectos Positivos o Negativos con el uso de la tecnología dentro de la Administración Pública}

La tecnología es un factor que ha modificado la realización de los actos jurídicos esto también son parte de la Administración Pública, teniendo ciertas ventajas la implementación tanto de las redes sociales, así como la manera de acceder a través de las plataformas diseñadas para realizar ciertos actos en que esto da agilidad de la presentación de la misma y la comodidad de no acudir a la Administración que corresponda.

A su vez también trae consecuencia desde forma negativa, pues como el acto ya no se realiza mediante un servidor público, sino por medio de un ordenador o sistema que en caso de tener una duda, no se puede acudir al servidor público que no está ateniendo, sino en algunas situaciones se puede tener ayuda por medio de un chat alterno de la página o a través de un número telefónico que se señale, pero a algunas personas esto le causa conflicto por no estar interactuando con una persona. La tecnología avanza constantemente a través del paso de los años pero esto no garantiza tener una seguridad de que la información proporcionada no sea utilizada de manera incorrecta. 
En materia fiscal cuando se cambio la obligación de realizar la contabilidad electrónica en México, se dio este tipo de inseguridad por parte de los contribuyentes, el uso de la tecnología a lo que se refiere el artículo 28 del Código Fiscal de la Federación que establece que esta se debería llevar los registro, asientos contables, así como el almacenamiento de esto a través de medios electrónicos, como la conservación de estos y lo que impacto es enviar la información de manera mensual cuando se tuviera la obligación de la información contable, es decir aquello referente a lo que son la balanza de comprobación, pólizas contables, catalogas de cuentas al órgano desconcentrado competente que en México es el Servicio de Administración Tributaria (SAT), siendo este un comienzo para solicitar la protección por medio de la garantía del amparo, esto se determinó por la Suprema Corte de Justicia de la Nación, señalando que el Anexo 24 de la Resolución Fiscal para el 2015 no eran adecuados debido a que no estaban en idioma español, así como no contar con los lineamientos técnicos para llevar a cabo este cambio, por eso se considero que se violaban los principios de legalidad y de seguridad hacia los contribuyentes, ya que tampoco eran emitidos por autoridades competentes quienes emitían dicha generación de archivos, a la fecha dichos motivos son solucionados con la reforma que se llevó acabo en el 2016 en noviembre al anexo antes citado para poder cumplir con los parámetros determinados por la Suprema Corte de Justicia de la Nación.

La cual ha sido superada mediante la tesis publicada en el Diario Oficial de la Federación en Abril de $2018^{4}$ la cual establece que el Anexo contemplado para la documentación electrónica no transfieren los derechos de los contribuyente por cumplir con todos los lineamientos que fueron solicitados, así como dieron todos los elementos para cumplir con la obligación formal que se consagra dentro del artículo 28 fracción IV del Código Fiscal de la Federación vigente, dando con esto una forma de cómo contemplar los requisitos, estableciendo así los lineamientos técnicos que no se habían contemplado en un principio.

Se contempla que la Administración Pública en la actualidad tenga en su dominio páginas de internet, en el caso de la Servicio de Administración Tributario por medio de la área correspondiente que es la Administración General de Comunicaciones y Tecnología de la Información (AGCTI), es la correspondiente de dar a conocer disposiciones de carácter general, no obstante que se publica de manera electrónica en la página oficial, no se deja la obligación que dichos lineamientos también sean publicado en el Diario Oficial de la Federación, siendo

${ }^{4}$ CONTABILIDAD ELECTRONICA. - EL ANEXO 24 DE LA RESOLUCIÓN MISCELÁNEA FISCAL PARA 2017, PUBLICADO EN EL DIARIO OFICIAL DE LA FEDERACIÓN EL 6 DE ENERO DE 2017, ACTUALIZA LOS SUPUESTOS A LOS QUE ESTABA SUJETA LA CONCESIÓN DEL AMPARO CONTRA DICHO ANEXO PARA 2015. [TA]; 10a. Época; 2a. Sala; Gaceta S.J.F.; Libro 53, Abril de 2018; Tomo I; Pág. 853. 2a. XXIV/2018 (10a.) 
esto un avance con respecto a la utilización de la tecnología pero teniendo la obligación de positivizar esa forma de cambio de la Administración por medio de la norma jurídica a través del proceso para la creación de la misma, es decir por medio del poder legislativo y ejecutivo mismo que se consagran dentro de los artículo 6 y 73 fracción XVII Constitucional para que la Administración pudiera utilizar la tecnología y dar conocer dicho lineamientos se tiene la facultad, pero a su vez se tiene que publicar en el Diario Oficial de la Federación lo concerniente en materia Federal y en la Gaceta Oficial aquello que corresponde a lo Estatal para cumplir con el principio de publicidad.

\section{TRANSPARENCIA DE LA INFORMACIÓN}

Con la apertura de las Secretarias para dar a conocer la información que tienen en sus archivos siempre que dicha información no sea considera como reservada o confidencial, este proceso se inicia con la norma jurídica contemplado dentro de la Ley Federal de Transparencia y Acceso de la Información para dar esa apertura hacia los gobernados, dar esa accesibilidad de tener la información que demandaran, pero a su vez esto fomentaría la transparencia de los actos por parte de la Administración, alguno de los objetivos sería combatir los actos irregulares de las Administraciones, así como también sería una forma de ejercer un Estado de Derecho Efectivo para los gobernados.

El cambio para una asequibilidad entorno al funcionamiento de la Administración pública electrónica dentro de la organización de sus gestiones para proveer de una manera oportuna los servicios y trámites que los gobernados necesitan, es como nace esto y a la facilidad de los medios electrónicos para ejercer dicha comunicación de una manera más dinámica y ese derecho a acceder a la información y desarrollar sistemas que maneje de una mejor manera los ingresos que percibe y el derecho patrimonial se efectúa de una manera más exitosa.

Este cambio tanto de la Administración como de los servidores que realizan dichas funciones deben cambiar hacia las tendencias de una digitalización dando con esto una entrega más efectiva de los actos de estos para realizar sus funciones y a su vez manejar la transparencia dentro de los recursos que le son asignados.

Da esta necesidad en México se implementó el organismo encargado de proteger ese derecho al acceso de la información que se denominaba Instituto Federal de Acceso a la Información Pública y Protección de Datos(IFAI), luego que se aprobara la Ley Federal de 
Transparencia y Acceso a la Información Pública Gubernamental, cambiando la denominación por Instituto Nacional de Transparencia, Acceso de la Información y Protección de Datos Personales (INAI), siendo un principio para la apertura de proporcionar información por parte del Ejecutivo y así también hacer que las solicitudes por parte de los ciudadanos tuviera respuestas acerca de la información que solicitaban al Estado, siempre que dicha información no fuera clasificada como reservada.

Con esto se ve un cambio de un Estado autoritario hacia un Estado democrático debido a la transición del uso de la información que se tiene a su alcance, haciendo modificaciones dentro de las actuaciones del Estado permitiendo tener un mayor control por parte de los ciudadanos en sus acciones y vigilándolos, generando una transformación hacia un gobierno transparente de sus actuaciones y dando un fortalecimiento a la democracia.

Siendo un comienzo para determinar que en efecto la publicidad es un punto importante para hacer que aquellos que tienen el poder puedan realizar de manera correcta y esto es una distinción entre las formas de gobiernos que son tiranas y aquellas que se quieren establecer como un República esto es considerado por Norberto Bobbio la importancia de la comunicación entre los actores que ejerce el gobierno para poder obtener un gobierno justo y democrático.

Generando con esto una libertad que se ha fundamentado de diversos actores tales como Michel Rosenfell ${ }^{5}$, quien determina lo siguiente:

1.-Dentro de un gobierno democrático que se determina por sus propias sus normas, es de suma importancia debido a que juega su rol dentro de su actuación como ente de poder y conforme a la opinión en cuestiones públicas y el acceso que tiene en la transparencia de sus actuaciones.

2.-El descubrimiento que torna hacia la verdad debido a la justificación de su facultad de expresar su voluntad como un sujeto que tiene capacidad jurídica dentro de su derecho individual como un sujeto de derecho.

Ahora bien, dentro de estas libertades se tiene que contemplar a la Administración Pública como un sujeto personal moral que se establece por organismos o instituciones que a su vez emiten opiniones acerca de los temas, así como manifiesta sus decisiones a través de los trámites que se le solicitan y dichas instituciones se configuran por personas físicas que son ellos quienes expresan ese derecho subjetivo de conformidad con la potestad que les confieren, por lo

\footnotetext{
${ }^{5}$ Citado por Fernández Rodríguez, José Julio "la modulación de la libertad de información en internet", Derechos Fundamentales y Estado, Instituto de Investigaciones Jurídicas, UNAM, México, 2002, p. 300
} 
tanto debe coincidir con la facultad que tiene dicho sujeto con la toma de decisión por parte del organismo que representa.

Esta apertura del cambio hacia la aplicación de la tecnología dentro de las Administraciones para existir una transparencia, da un cambio hacia un régimen democrático que se basa en un Estado de Derecho, siendo con esto una protección hacia los ciudadanos por ejercer su participación dentro de la gestión del gobierno, por medio de su colaboración y señalando con esto los limites correspondientes de las actuaciones pertinentes a los derechos subjetivos que les corresponden a los grupos sociales.

La exigencia de la transparencia de las actuaciones dentro de la Administración se realiza a través "de las exigencias constitucional de respeto a la ley como fundamento del sistema de derechos y del orden político. $Y$ sólo un régimen democrático ofrece, a través de la participación, garantías y oportunidades a los ciudadanos para que el ordenamiento jurídico

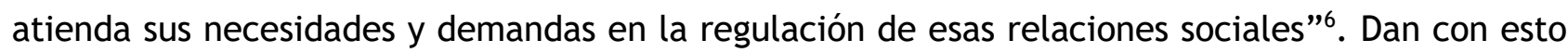
una limitación del exceso poder que pudiera llegar a percibir el Estado y siendo una contraposición de este el Derecho que se traduce en instituciones que son garantes del Derecho y protege el ordenamiento jurídico y sus actuaciones sean de forma uniforme, generales, de conformidad con el Derecho.

Esto parte de la primicia Constitucional que se otorga el derecho a tener un acceso a la información por medio de las garantías para generar un derecho y tener un buen funcionamiento dentro de la Administración y sus órganos y cumplir con el ordenamiento jurídico, siendo esta libertad el derecho a la información, que se tenga ese acceso a esta para ejercer la participación ciudadana en la toma de decisiones y cuestionar acerca de las acciones del Estado.

México ha tenido desde 1980 la oportunidad de establecer y ratificar Tratados Internacionales en los cuales se contemplan el Derecho a la Información como un Derecho Humano, tales como el Pacto Internacional de Derechos Civiles y Políticos que dentro de su artículo 19 fracción 2 establece: "Toda persona tiene derecho a la libertad de expresión; este derecho comprende la libertad de buscar, recibir y difundir informaciones e ideas de toda índole, sin consideración de fronteras, ya sea oralmente, por escrito o en forma impresa o artística, o por cualquier otro procedimiento de su elección"7, dando con esto un ideal a través del cual hombre pudiera alcanzar esa libertad y gozar de sus derechos tanto civiles como

\footnotetext{
${ }^{6}$ Ríos Estavillo Juan José, Derecho a la Información en México, Porrúa, México, 2005, Pág. 97

${ }^{7}$ http://www.ordenjuridico.gob.mx/TratInt/Derechos\%20Humanos/D47.pdf
} 
políticos y los Estados se obligan a actuar en favor de la comunicación y la transmisión de la información que está en su alcance con las limitaciones del mismo.

México se adhirió a dicho Pacto el 24 de marzo de 1981, y se promulgo a través de un Decreto en el Diario Oficial de la Federación con fecha del 20 de mayo de 1981 y en ese mismo año se publicó por medio de Diario Oficial de la Federación la Fe de Erratas el 22 de junio de 1981, dando comienzo a una nueva estructura dentro de nuestro país.

Dentro de la Convención Americana de Derechos Humanos, en su artículo número 13 se señala lo siguiente: "Toda persona tiene derecho a la libertad de pensamiento y de expresión. Este derecho comprende la libertad de buscar, recibir y difundir informaciones e ideas de toda índole, sin consideración de fronteras, ya sea oralmente, por escrito o en forma impresa o artística, o por cualquier otro procedimiento de su elección." 8 México adoptó dicho Pacto el 22 de noviembre de 1979, mismo que fue publicado en el Diario Oficial de la Federación el 19 de enero de 1981, considerando que el ideal de este pacto es referente a que el hombre tuviera esa libertad de derechos hacia el Estado para concebir instituciones democráticas que permitan concebir una justicia social y alcanzar con esta forma de organización el respeto de los derechos esenciales de los individuos que conforman los Estados.

Dando con esto que "el derecho a la información permite la pretensión de los ciudadanos frente a la actividad del Estado en determinadas prestaciones positivas, por ejemplo, el acceso a la información y documentación administrativa. En este sentido sería considerado un derecho social. Resulta así que desde esta clasificación el derecho a la

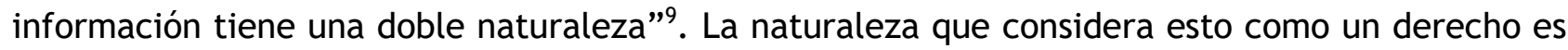
debido a que esa autoridad tiene la obligación de dar cumplimiento al derecho de la información, y en caso de que se haya perjudicado dicho derecho se pueda restituir.

Siendo que la autoridad tiene que respetar esos derechos de libertad de los ciudadanos desde la obligación de informar y proporcionar dicha información, generando este caso un derecho constitucional que se obtiene por tener una gestión con más transparencia y dar la información solicitada al sujeto que lo requiera, dando con esto una garantía para que el Estado incorpore este derecho y brinde una libertad hacia la información que no se encuentre dentro de una clasificación de reservado o confidencial, dando con esto una seguridad a ese derecho del acceso de la información.

\footnotetext{
${ }^{8}$ http://www.ordenjuridico.gob.mx/TratInt/Derechos\%20Humanos/D1BIS.pdf

${ }^{9}$ López Ayllón, Sergio, El Derecho a la Información, Miguel Ángel Porrúa, México, 1984, pág. 168
} 
Con esto se observa el principio de publicidad de los actos que manifiesta la autoridad a través de la administración y así los asuntos públicos de la gestión administrativa podrán lograr una transparencia de sus actuaciones, permitiendo tener un acceso de esa información a los gobernados.

Dando la referencia que dentro de la Administración Pública la información que se tiene puede versar acerca de aquella que tiene la característica de información pública que se puede acceder ya sea por medio de las paginas oficiales de la Administración o se tenga que publicar dentro de los medios correspondiente que la autoridad tenga que informar a los ciudadanos para que surta efecto dicha información o aquella que a solicitud de un individuo sea proporcionada la información que solicita por ese derecho que se tiene que se le otorgue siempre y cuando dicha información no sea clasificada como reservada o confidencial.

La información que se considera como confidencial es aquella comprendida en el artículo 110 de la Ley Federal de Transparencia y Acceso a la Información Pública, la cual señala como información reservada aquella que contengan los siguientes supuestos:

I. Comprometa la seguridad nacional, la seguridad pública o la defensa nacional y cuente con un propósito genuino y un efecto demostrable;

II. Pueda menoscabar la conducción de las negociaciones y relaciones internacionales;

III. Se entregue al Estado mexicano expresamente con ese carácter o el de confidencial por otro u otros sujetos de derecho internacional, excepto cuando se trate de violaciones graves de derechos humanos o delitos de lesa humanidad de conformidad con el derecho internacional;

IV. Pueda afectar la efectividad de las medidas adoptadas en relación con las políticas en materia monetaria, cambiaria o del sistema financiero del país; pueda poner en riesgo la estabilidad de las instituciones financieras susceptibles de ser consideradas de riesgo sistémico o del sistema financiero del país, pueda comprometer la seguridad en la provisión de moneda nacional al país, o pueda incrementar el costo de operaciones financieras que realicen los sujetos obligados del sector público federal;

V. Pueda poner en riesgo la vida, seguridad o salud de una persona física;

VI. Obstruya las actividades de verificación, inspección y auditoría relativas al cumplimiento de las leyes o afecte la recaudación de contribuciones;

VII. Obstruya la prevención o persecución de los delitos;

VIII. La que contenga las opiniones, recomendaciones o puntos de vista que formen parte del proceso deliberativo de los Servidores Públicos, hasta en tanto no sea adoptada la decisión definitiva, la cual deberá estar documentada;

IX. Obstruya los procedimientos para fincar responsabilidad a los Servidores Públicos, en tanto no se haya dictado la resolución administrativa;

$X$. Afecte los derechos del debido proceso;

XI. Vulnere la conducción de los Expedientes judiciales o de los procedimientos administrativos seguidos en forma de juicio, en tanto no hayan causado estado;

XII. Se encuentre contenida dentro de las investigaciones de hechos que la ley señale como delitos y se tramiten ante el Ministerio Público, y 
XIII. Las que por disposición expresa de una ley tengan tal carácter, siempre que sean acordes con las bases, principios y disposiciones establecidos en la Ley General y esta Ley y no las contravengan; así como las previstas en tratados internacionales. ${ }^{10}$

Dentro de esa misma disposición se señala que se entiende por información confidencial aquella que:

I. La que contiene datos personales concernientes a una persona física identificada o identificable;

II. Los secretos bancario, fiduciario, industrial, comercial, fiscal, bursátil y postal, cuya titularidad corresponda a particulares, sujetos de derecho internacional o a sujetos obligados cuando no involucren el ejercicio de recursos públicos, y

III. Aquella que presenten los particulares a los sujetos obligados, siempre que tengan el derecho a ello, de conformidad con lo dispuesto por las leyes o los tratados internacionales. ${ }^{11}$

Dentro de la información antes señalada, se establece que no se encuentra sujeta a un plazo de tiempo y solamente se puede acceder a esa información por los titulares, los representantes o los servidores públicos.

El periodo que una información clasificada como reservada es de cinco años, no obstante es posible ampliar este periodo por otros cincos años, siempre y cuando se fundamente y se justifique con el motivo que originalmente brindó el comité, así como las razones para incorporarla dentro de esta clasificación.

En caso de que la información con carácter reservado se trate de una violación de los derechos humanos con una condición de grave o se trate del delito de lesa humanidad o ya sea que dicha información se encuentre relacionados con delitos relacionados a actos de corrupción entonces por estas razones se podrá conocer de dicha información debido a que los motivos señalados son un derecho subjetivo público que nos compete para aclarar dichas situaciones.

Dicho lo anterior se va visualizando la implementación de la tecnología dentro de la Administración Publica y del Derecho Administrativo siendo un inicio del estudio tanto desde el aspecto científico con el jurídico a través del cual la información que se obtiene puede considerarse como reservada o pública siendo esto una determinación de acuerdo a las normatividades para ejercer ese derecho a la información.

Es así como a través de los principios Constitucionales que existen en México que dentro de su artículo número 6 se ha implementado ese cambio hacia el uso de la tecnología dentro de

${ }^{10} \mathrm{http}: / /$ www.diputados.gob.mx/LeyesBiblio/pdf/LFTAIP_270117.pdf, artículo 110

11 http://www.diputados.gob.mx/LeyesBiblio/pdf/LFTAIP_270117.pdf, artículo 113 
ese acceso a la información dando con esto una apertura de cambios para ayudar a que la Administración pueda tener una mejor distribución de sus actividades y un contacto más cercano con los ciudadanos, por lo tanto el derecho de acceso a la información "radica también en una serie de obligaciones, sobre todo de carácter formal y normativas, que debe sr cumplimentadas por todos aquellos que utilicen la vía o referentes implementados en una norma jurídica". ${ }^{12}$ Siendo esto una forma de tener un verdadero Derecho al acceso de la información y no solamente que se quede como un matiz de Políticas Públicas por parte de la Administración.

Es así como este cambio de la tecnología se va desarrollando, reestructurando la forma de ejercer los actos administrativos y realizar con esto una nueva forma de criterio en la emisión de los mismos, para asentar lo más efectivo que sería para la eficacia de la prestación de la Administración a la comunidad y legitimar el uso de la nueva tecnología para facilitar los procesos que lleva consigo.

Es así como se va cambiando a raíz de que la sociedad empieza ejercer un poder hacia el Estado y con ello se da una limitación del poder y un equilibrio en la relación jurídica entre el Estado y los gobernados. A través de los medios electrónicos las Administraciones tienen un control más inmediato y amplio de sus procesos dentro de sus facultades pero a la vez dicha información podrá ser solicitada el gobernando para saber si se está cumpliendo de manera correcta con los ingresos percibidos por el Estado. También se habla acerca de esta tutela como protección que a través de la libertad informática ha influido para que los ciudadanos empiecen a ejercitar sus derechos y tengan esa facultad de réplica para exigir a las Administraciones que realicen los procesos como deben efectuarse.

Ahora bien, esto es un punto de equilibrio también debido a que las Administraciones buscan tener una eficacia administrativa, pueden utilizar la facilidad de la tecnología para lograr que dentro de sus procesos se eficienticen y lograr contar con una buena técnica en beneficios de todos y con esto ayudar a tener una buena Administración. 3 Principios que debe contar la Administración Pública Electrónica

${ }^{12}$ Ríos Estavillo Juan José. Derecho a la Información en México. Porrúa, México, 2005. p. 137 


\section{PRINCIPIOS QUE DEBE CONTAR LA ADMINISTRACIÓN PÚBLICA ELECTRÓNICA}

Dentro de este apartado se estiman aquellos principios que se deben prever dentro de las actuaciones de la Administración para ejercer sus actos de manera eficiente y eficaz enumerando los siguientes ${ }^{13}$ :

Se concibe el principio de la legalidad como aquel concerniente a la implementación de la norma jurídica que señale la utilización de los medios electrónicos en particular de la Administración Pública y de los gobernados para hacer uso de su manifestación de voluntad en emitir actos jurídicos.

Siendo importante someter el principio de la igualdad que concibe que el acceso de los medios electrónicos para dirigirse a la Administración sea igual para todos desde el punto de vista de accesibilidad, ya que algunos se puede dificultar la manera de cómo ingresar a lo misma.

Los principios de eficacia y eficiencia que consagra la Administración deben aplicarse en el uso de la tecnología que sea más adecuada para atender a las necesidades de los gobernados y tener un acceso sencillo y oportuno al mismo para facilitar el uso dentro de sus páginas electrónicas, así como el principio de responsabilidad de la Administración Pública debe resguardar la información y los datos que tenga a su disposición ya sea por escrito o electrónicos, con la reserva de aquella información que sea clasificada como confidencial para no externar sus archivos o datos a persona que no solicite la misma.

En México el principio de transparencia se observa a partir del año 2000, cuando se pretendió tener un contacto más directo y oportuno con los gobernados y a través del cual la Administración Pública mediante el uso de la tecnología se hizo más confiable la difusión, la publicidad de sus actuaciones, como sus cuentas o compras a través de los sistemas que utiliza para proporcionar la información de esto a los gobernados.

Se debe establecer la garantía de conservar la comunicación con los destinarios al mismo tiempo de resguardar y no dar comunicación a personas que no estén dentro del proceso

13 "Carta Iberoamericana de Gobierno Electrónico" de Pucón (Chile) de $1^{\circ}$ de junio de 2007 (aprobada en la IX conferencia Iberoamericana de Ministros de administración pública y Reforma del Estado). 
y dar autenticidad de los sujetos que intervienen dentro de los procesos de participación con las Administraciones.

Las Administraciones deben procurar que la accesibilidad de la información, así como de aquellos servicios que puedan realizar por medios electrónicos, sea a través de un diseño que sea amigable y fácil de utilizar, que los canales de estos sean accesibles y que tengan el soporte correcto para evitar fallas en dichas páginas para que puedan tener un acceso correcto a estas.

Con esto se establece el principio de la simplificación de los trámites que se realizan a través del ejercicio de la función administrativa, y con ello se logren suprimir ciertos requisitos, debido a que ya lo tiene dentro de sus archivos de las administraciones y así reducir tanto los documentos que se piden como el plazo para contestar por parte de la Administración, con lo que se logra tener una simplificación dentro del mismo.

\section{LA EFECTIVIDAD DEL ACTO ADMINISTRATIVO EN LA VÍA TECNOLÓGICA}

Debido a las diversas funciones y atribuciones que tiene la Administración Publica para cumplir con los fines del Estado, siendo esta una relación jurídica por eso los efectos que emite la Administración por sus diversos órganos debe estar regulados por el Derecho para que surta efecto los actos jurídicos debido a que este se realizan con la intención de la voluntad del órgano de la Administración para producir consecuencias de Derecho.

Cuando la Administración realiza actos que no produce consecuencias de derecho se realiza para comunicar algo que no tiene una implicación, se en los casos que se una invitación para atender algo, cuestiones generales para comunicarle algo o recomendaciones esto no implica un efecto jurídico, siendo que esto se le puede tornar como hechos jurídicos debido a que no se tiene la intención de producir una consecuencia de derecho.

En cambio, los actos jurídicos que emite la administración esto radica que tiene la manifestación de la voluntad de producir consecuencias de derecho por lo tanto pueden crear, transformar, modificar o extinguir ya sean derechos u obligaciones, a través de ya sea de actos administrativos, reglamentos administrativos, concesiones, contratos administrativos.

Surge ahora le necesidad de ese acto administrativo que produce efecto por parte de la Administración Pública tenga dentro de sus características la implementación de la tecnología para que surta efectos de ahí la importancia de este cambio de paradigma. 


\subsection{Conceptualización del Acto Administrativo}

La administración publica no recae todas sus actuaciones a través del acto administrativo debido a que ocasione emiten actos de administración, la diferencia radica en la consecuencia de producir efectos jurídicos.

Por lo tanto, podemos externar que el acto administrativo se considera como la “declaración unilateral y concreta del órgano ejecutivo que produce efectos jurídicos directos e inmediatos" 14 en cambio en la Ley Federal de Procedimiento Administrativo marca cuales son los elementos y requisitos del acto administrativo siendo los siguientes:

I. Ser expedido por órgano competente, a través de servidor público, y en caso de que dicho órgano fuere colegiado, reúna las formalidades de la ley o decreto para emitirlo;

II. Tener objeto que pueda ser materia del mismo; determinado o determinable; preciso en cuanto a las circunstancias de tiempo y lugar, y previsto por la ley;

III. Cumplir con la finalidad de interés público regulado por las normas en que se concreta, sin que puedan perseguirse otros fines distintos;

IV. Hacer constar por escrito y con la firma autógrafa de la autoridad que lo expida, salvo en aquellos casos en que la ley autorice otra forma de expedición;

V. Estar fundado y motivado;

VI.- (Se deroga)

VII. Ser expedido sujetándose a las disposiciones relativas al procedimiento administrativo previstas en esta Ley;

VIII. Ser expedido sin que medie error sobre el objeto, causa o motivo, o sobre el fin del acto;

IX. Ser expedido sin que medie dolo o violencia en su emisión;

$X$. Mencionar el órgano del cual emana;

XI.- (Se deroga)

XII. Ser expedido sin que medie error respecto a la referencia específica de identificación del expediente, documentos o nombre completo de las personas;

XIII. Ser expedido señalando lugar y fecha de emisión;

XIV. Tratándose de actos administrativos deban notificarse deberá hacerse mención de la oficina en que se encuentra y puede ser consultado el expediente respectivo;

XV. Tratándose de actos administrativos recurribles deberá hacerse mención de los recursos que procedan, y

XVI. Ser expedido decidiendo expresamente todos los puntos propuestos por las partes o establecidos por la ley. ${ }^{15}$

Ahora bien, analizando los actos administrativo desde la concepción de actos administrativos a través de la digitalización se puede corroborar que en la fracción número IV

\footnotetext{
${ }^{14}$ Diez, Manuel María. Manual de Derecho Administrativo. Dos volúmenes, Plus-Ultra, Buenos Aires, 1983, pág. 178

${ }^{15}$ http://www.diputados.gob.mx/LeyesBiblio/pdf/112_180518.pdf, artículo 3
} 
señala que el acto administrativo debe constar por escrito y con firma autógrafa de autoridad que lo expida sin mencionar el uso de los medios de la tecnología para emitir dicho actos, solamente se puede observar que se mencione en dichas normas la forma de la utilización de la misma, es aquí el problema de contar con medios tecnológicos pero que no se puede aplicar para emitir actos administrativos debido a que no están regulado por la norma.

Se señala que la tecnología facilita la actuación de las Administraciones, pero hasta que no esté regulado se podrá usar este medio, hasta que sea permitido por medio de la norma jurídica para emitir actos administrativos electrónicos, así como implementar el uso de la gestión pública por medio electrónicos que sirvan para cumplir con los fines del Estado.

Los elementos que se consideran requisitos para emitir un acto administrativo consideran que le dan validez y forma de la manifestación de la autoridad que quiere expresar al gobernado, dando esa validez al acto debido a que tenga competencia y manifieste su voluntad la persona que se asigna a la función de dicho órgano de la administración para cumplir con los requisitos y elementos para que se constituya como un acto administrativo.

Los objetivos del acto administrativo sirven para expresar de manera clara la resolución de la Administración al acto solicitado por el gobernado, señalando que dentro del articulo antes mencionado no se hace diferencia entre cuáles son los elementos del acto administrativo y cuáles son los requisitos, dando con esto un numeral extenso de lo que debe contener el acto administrativo.

Los requisitos del acto administrativo son aquellos que tienen por objeto señalar la eficacia y validez del acto administrativo, siendo que esto es lo que determina ya sea la competencia de la autoridad, el objeto del acto administrativo, motivo y fin del mismo, cuáles es la forma, manera de la publicación, así como el procedimiento y las causas del mismo, teniendo así que los elementos serían en este caso el sujeto que emite dicho acto administrativo, su voluntad para emitirlo y por último el precepto que determina ese acto administrativo.

Si bien se da el uso de la tecnología dentro de la Administración Pública aun en la normatividad no se contempla la posibilidad de externar por medios electrónicos los actos administrativos tendientes a emitir la decisión de las autoridades, con excepción de aquellos que son permitidos por alguna norma jurídica como lo son la auditorias que se emite por medios de electrónicos en materia fiscal, pero aún no se tiene la matización de la emisión electrónica de los actos administrativos. 
Las Administraciones han aplicado el uso de la tecnología para agilizar y tener el orden del control de ciertas áreas, como aquellas que llevan un proceso de ingresar la información, archivarla y contar con base de datos que estén actualizados, como los registros de cuenta, ya sea prediales, personas, etc.

La importancia de que dentro de la norma jurídica se especifique que dichos actos se podrán llevar a cabo mediante la digitalización, con los requisitos y elementos que consten para emitir los actos administrativos y tener eficacia y validez y así dar un adelanto a un gobierno de tecnología y poder avanzar dentro del concepto de la Buena Administración y disminuir con esto los recursos que se necesitan dentro de las Administraciones cumpliendo con los principios y derechos que se deben percibir dentro del Estado.

\section{CONCLUSIÓN}

El gobierno digital es una necesidad dentro del cambio que estamos teniendo como sociedad con el uso de la tecnología, por lo que se tiene que contemplar que los actos administrativos deben de tender a realizarse de manera electrónica, tanto como por cuestiones de cuidado al medio ambiente, así como eficacia de los mismos, por lo que se debe cambiar la normatividad para permitir este proceso y que se estructure de una manera adecuada la legitimidad de este derecho.

Aunque en México algunas secretarias emiten ciertos actos de manera electrónica tales como la Secretaría de: Función Pública; Hacienda y Crédito Público; Economía; Comunicación y Transporte; Relaciones Exteriores, siendo esto un principio para mirar este cambio aun no hay una legislación que contemple la utilización de los documentos electrónicos como una forma de emitir los actos administrativos.

El uso de tecnologías ha permitido la simplificación de algunos trámites y de igual manera permite que el acceso a los archivos de las Administraciones sean más accesibles, pero debido a la política pública interna y a que no hay una reglamentación adecuada para llevar a cabo la regulación del derecho electrónico en la actuación de la Administración Pública, se constituye un desafío tanto para los legisladores y el Ejecutivo en cambiar hacia este medio, los cambios son a veces molestos pero logran ventajas que a larga traen consecuencias positivas. Teniendo el compromiso de tener una Buena Administración, y teniendo como principio "respetar los principios de transparencia y acceso a la información, legalidad, imparcialidad, 
igualdad de trato"16 se debe de procurar una sociedad justa a través de la democracia y ejerciendo un Derecho acorde a las necesidades reales de la sociedad.

\section{REFERENCIAS}

DIEZ, Manuel María. Manual de Derecho Administrativo. Dos volúmenes, Plus-Ultra, Buenos Aires, 1983.

FERNÁNDEZ RODRÍGUEZ; José Julio. La modulación de la libertad de información en internet. In: Derechos Fundamentales y Estado, Instituto de Investigaciones Jurídicas. UNAM, México, 2002.

GALINDO CAMACHO, Miguel. Teoría de la Administración Pública. Porrúa, México, 2000 KAPLAN, Marcos. Ciencia, Estado y derecho en la tercera Revolución. Universidad Autónoma de México, 2000, México.

LÓPEZ AYLLÓN, Sergio. El Derecho a la Información. Miguel Ángel Porrúa, México, 1984

González Sanmiguel, Nancy Nelly. La Influencia de los Derechos Humanos dentro de la justicia Administrativa en México. Tirant lo Blanch, México, 2018.

RÍOS ESTAVILLO, Juan José. Derecho a la Información en México. Porrúa, México, 2005.

CONTABILIDAD ELECTRONICA. - EL ANEXO 24 DE LA RESOLUCIÓN MISCELÁNEA FISCAL PARA 2017, PUBLICADO EN EL DIARIO OFICIAL DE LA FEDERACIÓN EL 6 DE ENERO DE 2017, ACTUALIZA LOS SUPUESTOS A LOS QUE ESTABA SUJETA LA CONCESIÓN DEL AMPARO CONTRA DICHO ANEXO PARA 2015. [TA]; 10a. Época; 2a. Sala; Gaceta S.J.F.; Libro 53, Abril de 2018; Tomo I; Pág. 853. $2 a$. XXIV/2018 (10a.)

http://www.diputados.gob.mx/LeyesBiblio/pdf/LFTAIP_270117.pdf

Carta Iberoamericana de Gobierno Electrónico" de Pucón (Chile) de $1^{\circ}$ de junio de 2007 (aprobada en la IX conferencia Iberoamericana de Ministros de administración pública y Reforma del Estado).

http://www.diputados.gob.mx/LeyesBiblio/pdf/112_180518.pdf

http://www.ordenjuridico.gob.mx/TratInt/Derechos\%20Humanos/D47.pdf

http://www.ordenjuridico.gob.mx/TratInt/Derechos\%20Humanos/D1BIS.pdf

ARTIGO CONVIDADO / Publicado em 02.10.2019

${ }^{16}$ González Sanmiguel, Nancy Nelly, La Influencia de los Derechos Humanos dentro de la justicia Administrativa en México, Tirant lo Blanch, México, 2018, Pág. 161 


\section{COMO FAZER REFERÊNCIA AO ARTIGO (ABNT/BRASIL):}

GONZÁLEZ SANMIGUEL, Nancy Nelly. El derecho protección y la regulación del uso de las nuevas tecnologías desde el Derecho Administrativo. Revista Eletrônica do Curso de Direito da UFSM, Santa Maria, RS, v. 14, n. 1, e40341, jan./abr. 2019. ISSN 1981-3694. DOI: http://dx.doi.org/10.5902/1981369440341. Disponível em:

https://periodicos.ufsm.br/revistadireito/article/view/40341 Acesso em: dia mês. ano.

Direitos autorais 2019 Revista Eletrônica do Curso de Direito da UFSM

Editores responsáveis: Rafael Santos de Oliveira e Angela Araujo da Silveira Espindola

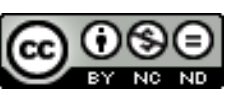

Este obra está licenciado com uma Licença Creative Commons Atribuição-NãoComercial-SemDerivações 4.0 Internacional.

\section{SOBRE A AUTORA}

Nancy Nelly González Sanmiguel

Profesora de tiempo completo en la Facultad de Derecho y Criminología de la Universidad Autónoma de Nuevo León. Doctora en Derecho. 